\title{
Ultrafast gated imaging of laser produced plasmas using the optical Kerr effect
}

D. R. Symes, U. Wegner, H-C. Ahlswede, M. J. V. Streeter, P. Galegos, E. J. Divall, P. P. Rajeev, D. Neely, R. A. Smith

1. Central Laser Facility, Rutherford Appleton Laboratory, Chilton, Oxon, OX11 OQX, United Kingdom

2. Blackett Laboratory, Imperial College of Science, Technology and Medicine, Prince Consort Road, London SW7 2BZ, United Kingdom

\begin{abstract}
Optical imaging is a versatile diagnostic for investigations of plasmas generated under intense laser irradiation. Gating techniques are commonly used to reduce the amount of light detected from the self-emission of the hot plasma or to improve the temporal resolution of the detector. The use of an optical Kerr gate enables a superior dynamic range and temporal resolution compared to electronically gated devices. The application of this method for the imaging of laser produced plasmas is demonstrated, and the possibility to produce a sub-10fs, high dynamic range streak camera is discussed.
\end{abstract}

This paper was prepared for submittal to Applied Physics Letters 


\section{Introduction}

Optical probing is a powerful tool for diagnosing high energy density plasmas generated using intense laser pulses, enabling measurement of the electron density [1] and visualization of energy transport and shock propagation [2]. Ultrafast $(<100 \mathrm{fs})$ laser pulses can be employed as a backlighter for transverse imaging, providing twodimensional snapshots of the interaction with a temporal resolution limited only by the transit time of the pulse through the plasma. The emission of light directly from the interaction can also be imaged: both the radiation from the hot laser-heated plasma which lasts of order nanoseconds [GOI] and also short bursts of transition radiation emitted as relativistic electrons leave the target [OTR]. The resolution for these measurements is determined by the exposure time of the camera, which can be electronically gated to be on the timescale of picoseconds. A streak camera can be used to record one-dimensional information as a function of time, with the temporal resolution of electronically streaked instruments also being typically of order a picosecond. Temporal gating has the additional benefit that the exposure time can selected so that the detector is blind to the initial flash of self-emission from the plasma. When backlighting plasmas without gated detectors this light contributes a significant background level and necessitates a high brightness in the probe pulse.

The exposure time of the detector can be further reduced if the gate is activated optically rather than electronically. This can be achieved by incorporating into the imaging system an optical Kerr gate $(\mathrm{OKG})$ composed of two crossed polarizers around a non-linear medium [5]. The gate is opened by passing an intense laser pulse through the gating medium to induce a birefringence. The polarization rotation introduced to the probe light allows it to transit the second polarizer to reach the detector. This gating technique has been used for applications such as Raman and fluorescence spectroscopy [6-9] and ballistic-photon imaging [10-12]. The OKG is also the basis for polarization gated frequency-resolved optical gating (PG-FROG) devices used to diagnose laser pulses [13]. In this case fused silica (FS) is chosen as the gating material because its nonlinear response is effectively instantaneous and so the FROG can measure ultra-short pulse durations (<10fs). In other applications liquid carbon disulfide is commonly used because of its strong non-linear response $\left(n_{2}=3.1 \times 10^{-14} \mathrm{~cm}^{2} \mathrm{~W}^{-1}\right) . \mathrm{CS}_{2}$ has a slow relaxation time of $\sim 1$ ps because it is dominated by molecular reorientation rather than a purely electronic response. FS is thus superior in terms of temporal response but it has a much lower non-linear refractive index $\left(n_{2}=2.6 \times 10^{-16} \mathrm{~cm}^{2} \mathrm{~W}^{-1}\right)$ and so higher intensities are needed to obtain the polarization rotation. An investigation into the properties of various glasses and crystals with the aim of sourcing a material with both strong and ultrafast non-linear response has been reported [7,8].

In this paper we apply the optical gating method to the imaging of laser-produced plasmas. We use a short pulse back-lighter to provide shadowgraphic images of the laser interaction and demonstrate the suppression of background light from the plasma selfemission. With further optimization this system could provide two-dimensional images with sub-10fs temporal resolution, limited only by the duration of the pulse that drives the gate, and an exceptional dynamic range determined by the extinction ratio of the polarizers $\left(\sim 10^{-5}\right)$. Furthermore, an ultrafast $(<50 \mathrm{fs})$ high dynamic range all-optical streak camera could be produced by tilting the gate pulse with respect to the probe as used in 
FROG measurements [13]. Such an arrangement has also been reported using the linear electro-optic effect to measure terahertz pulses $[14,15]$.

\section{Experiment}

The gated imaging scheme comprised a four lens system which image relayed the plasma interaction to the position of the Kerr gate and then re-imaged with magnification to the charge-coupled device (CCD) camera. A linearly polarized probe beam was used as a backlighter, which was split before the CCD and focused onto a photodiode to monitor gating performance. Short pass filters were placed in front of both the CCD and the photodiode to block light at the fundamental $800 \mathrm{~nm}$ wavelength. The gating beam was aligned collinearly with the probe through the gating position using dichroic mirrors which transmitted the probe beam. The OKG was then formed by placing a non-linear material at the gate position and a polarizer after the gate rejection mirror. In some cases, a polarizer was added before the gate to optimize the probe polarization. The gating beam passed a half-waveplate to orient its polarization at $45^{\circ}$ to the probe and was focused through the $\mathrm{OKG}$ in order to reach sufficient intensity to activate the gate. However, the gating beam size needed to remain large enough to provide an adequate field of view for plasma imaging.

The experiment was performed using the output from a differentially pumped gas filled hollow fiber pulse compressor (HFPC) [16] injected with low-energy $(<5 \mathrm{~mJ})$ pulses split from the Astra laser system. When optimized, a HFPC can provide broadband pulses with energies $>5 \mathrm{~mJ}$ and pulse durations below 10fs [17]. In the current experiment we operated the fiber without a gas fill so there was no spectral broadening and the pulse duration remained at $\sim 60 \mathrm{fs}$. The pulse emerging from the fiber had an energy $\sim 1 \mathrm{~mJ}$ and a smooth spatial profile with diameter $\sim 7 \mathrm{~mm}$. This beam passed through a thin $\beta$-BariumBorate crystal to provide a second harmonic $(400 \mathrm{~nm})$ probe pulse and the fundamental beam was used as the gating pulse. At the OKG the gating beam had an energy $\sim 0.5 \mathrm{~mJ}$ in a spot of $1 \mathrm{~mm}$ diameter generating an intensity $\sim 2.5 \times 10^{11} \mathrm{Wcm}^{-2}$.

We investigated the properties of the gated imaging system by measuring the transmitted 400nm signal measured on the photodiode as a function of the delay of the gating beam. To remove any background from probe light unaffected by the gating process, the size of the probe beam at the OKG was made smaller than that of the gating beam. We tested two glasses as gating materials: FS and Schott NSF-66. These samples were 1 inch diameter windows with thickness $L=1 \mathrm{~mm}$ and the FS had a broadband antireflection coating on both sides. Both glasses are inexpensive and FS is well known to have an instantaneous ( $1 \mathrm{fs})$ response [13]. NSF-66 is a high-index glass (1.914 at $632.8 \mathrm{~nm}$ ) which was chosen because Kinoshita et al found that the gating effect is stronger with increasing linear refractive index [7].

\section{Gate characterisation}

The transmitted signal as a function of the intensity of the gating beam is shown in the inset to Fig. 1 for FS. The Kerr effect becomes significant once the intensity exceeds $8 \times 10^{10} \mathrm{Wcm}^{-2}(150 \mu \mathrm{J})$ and follows a quadratic dependence with increasing gate beam 
energy (as seen in Ref. 5) until $\sim 2.5 \times 10^{11} \mathrm{Wcm}^{-2}(500 \mu \mathrm{J})$ when the dependence becomes weaker. At this point the transmission efficiency of the gate is $\sim 20 \%$; with the polarizers open the transmitted signal is $\sim 1.4 \mathrm{~V}$. This transmission seems reasonable when we consider that the intensity required to drive a half-wave rotation of the probe beam polarization is $I_{\lambda / 2}=\lambda /\left(2 n_{2} L\right) \sim 7.5 \times 10^{11} \mathrm{Wcm}^{-2}$. When we used NSF-66 as the gating material, the maximum gating beam intensity was limited by the onset of white light generation in the glass. This was clearly seen as a large increase in signal on the diode and complete saturation of the CCD camera. At this intensity, the efficiency of NSF-66 was only slightly higher $(\sim 23 \%)$ than the FS.

The temporal response of the two glasses is shown in Fig. 1 where the transmitted signal is plotted against the gating beam delay. In NSF-66 the birefringence is maintained over a long period of $\sim 1.5 \mathrm{ps}$, which is comparable to $\mathrm{CS}_{2}$, precluding ultrafast gating. In contrast the signal measured in the FS gate is fitted with a Gaussian with a full width half maximum of $180 \mathrm{fs}$. For ultrafast gating, this measurement does not yield a value for the gate duration because if the material response time and the gating pulse duration are both shorter than the probe pulse duration then the signal width is determined by the latter. In our current layout the probe duration was stretched because of transit through two dichroic mirrors. In future work we intend to conduct a more sophisticated measurement of the gate duration. However, given that FS is known to have a femtosecond response time we expect the duration of the gate to be limited only by the gate duration which is $\sim 60 \mathrm{fs}$.

We investigated the dynamic range of the system by splitting the probe pulse in a Michelson interferometer and attenuating one arm with a filter of optical density [ND31x2]. Figure 2 shows a scan of the gating beam delay with the zero position defined as the peak of the weaker pulse. The attenuated pulse could easily be resolved in the absence of the stronger pulse (at $7.5 \mathrm{ps}$ ), when the background level was negligible. With the stronger pulse present, the background level increased to $\sim 40 \mathrm{mV}$ (dashed line) almost obscuring the weak pulse. This background at a level of $\sim 7 \%$ is caused by leakage of the strong pulse through the crossed polarizers which, in this arrangement, had an extinction ratio of 100. With improved optimization of the polarizer pair, extinction ratios of order $10^{-5}$ should be achievable rendering the background negligible and greatly enhancing the dynamic range of the instrument.

\section{Plasma imaging}

The next part of the experiment was to demonstrate that the Kerr gating method can be used to prevent the bright emission from hot plasma blinding the detector. For high laser irradiances this background is present on every shot because of the extreme temperatures generated. At the moderate intensities used here, the plasma emission from a single shot is not bright compared to the brightness of the probe beam. However, we can produce a large amount of emission by using multiple laser shots, because the complicated structure of the damage crater increases the laser absorption efficiency [18]. Hence, this can be used to mimic the situation encountered on a high intensity laser shot.

We used the gated imaging system to measure shadowgraphic images of the plasma formed from the irradiation of a glass target of $100 \mu \mathrm{m}$ thickness. The plasma production laser had an energy of $\sim 2 \mathrm{~mJ}$, a pulse duration $\sim 60 \mathrm{fs}$ and was focused to a spot 
of diameter $70 \mu \mathrm{m}$ generating an intensity $\sim 2.5 \times 10^{14} \mathrm{Wcm}^{-2}$. Every image was recorded while the target was irradiated with a series of 100 shots at a repetition rate of $10 \mathrm{~Hz}$. Figure 3 shows the target after 10 (left) and 100 (right) shots with the time delay between the drive pulse and the gated probe pulse set at 2.5ns. Without optical gating (Fig. 3a), plasma emission can be occasionally seen after $\sim 5$ shots and becomes problematic after $\sim 20$ shots. This is increasingly obstructive with further laser shots until the entire interaction region is obscured as plasma emission saturates the camera. In contrast, when the optical gating is active (Fig. 3b) no plasma emission is visible. This allows clear images to be taken of the structure of the glass throughout the shot series and enables diagnosis of the emergence and propagation of cracks and the opaquing of the crater. After 200 shots the drive laser drills through the target and the probe illumination can again be seen at the centre of the damage site (not shown).

Images of a wire taken using two alternative gating materials are shown in Fig. 4. We obtained clear images with a $500 \mu \mathrm{m}$ thick zinc oxide wafer, a material used for gated Raman microscopy [19], but gating was not possible because the 400nm probe pulse was absorbed in the presence of the intense gating pulse. This could be solved by using a different probe wavelength or using a different crystalline material [8]. We also tested a sample of lead-bismuth-gallium glass which has previously been used to create a fast Kerr gate [20] but the poor optical quality of the glass leads to striations in the image and so it cannot be used for imaging applications.

\section{Conclusion}

To conclude, we have demonstrated that optical Kerr gating can be incorporated into the imaging systems commonly used for diagnosing high energy density plasmas created using intense laser pulses. We presented images of multiple pulse irradiation of glass targets which show the effective elimination of plasma self-emission from the camera. This is of great benefit for experiments using ultra-intense laser systems in which this background constitutes a common problem. These images also highlight the potential of the optical gating technique for investigations of material properties, such as laser optic damage testing [21,22]. Rajeev - any more material refs?

The Kerr gate was driven in fused silica with the output from a hollow fiber pulse compressor. With further optimization the gate should be operable on a sub-10fs timescale. For such ultrafast instrument response a careful choice of components is required as considered in Refs. [8,9]. In particular, the polarizer pair must have low dispersion, which can be achieved by using thin sheet $(<0.5 \mathrm{~mm})$ or wire-grid $(<1 \mathrm{~mm})$ polarizers. These also exhibit high extinction ratios $\left(10^{3}-10^{4}: 1\right)$ which will enable a reduction in the background light level improving contrast. Alfano et al. [10] have reported Kerr gated imaging of droplets within a turbid medium with a signal-to-noise ratio of $10^{-10}$ [CHECK THIS]. Adding a tilt to the gating beam akin to the FROG arrangement [13] will convert the device to an ultrafast streak camera. These instruments will therefore constitute low-cost plasma diagnostics with superior temporal resolution and dynamic range properties compared to conventional electronic devices.

\section{Acknowledgements}


We acknowledge useful discussions with P. Matousek, J. Collier, I. Tracey, K. Ronayne, U. Teubner, E. Gumbrell, S. Mangles and J. Underwood. We thank the Astra laser operators and target fabrication team. This research was partly funded by CLIK.

\section{References}

[1] I. H. Hutchinson, Principles of Plasma Diagnostics (Cambridge University Press, Cambridge, 1987).

[2] B. A. Remington, R. P. Drake, and D. D. Ryutov, Rev. Mod. Phys. 78, 755 (2006).

[3] GOI ref

[4] J. J. Santos, F. Amiranoff, S. D. Baton, L. Gremillet, M. Koenig, E. Martinolli, M.

Rabec Le Gloahec, C. Rousseaux, D. Batani, A. Bernardinello, G. Greison, and T. Hall, Phys. Rev. Lett. 89, 025001 (2002).

[5] P. P. Ho and R. R. Alfano, Phys. Rev. A 20, 2170 (1979)

[6] P. Matousek, M. Towrie, A. Stanley, and A. W. Parker, Appl. Spectrosc. 53, 1485 (1999).

[7] S. Kinoshita, H. Ozawa, Y. Kanematsu, I. Tanaka, N. Sugimoto, and S. Fujiwara, Rev. Sci. Instrum. 71, 3317 (2000).

[8] R. Nakamura and Y. Kanematsu, Rev. Sci. Instrum. 75, 636 (2004)

[9] B. Schmidt, S. Laimgruber, W. Zinth, and P. Gilch, Appl. Phys. B 76, 809 (2003).

[10] R. R. Alfano, X. Lian, L. Wang, and P. P. Ho, Science 264, 1913 (1994).

[11] C. Dunsby and P. M. W. French, J. Phys. D: Appl. Phys. 36, R207 (2003).

[12] J. B. Schmidt, Z. D. Schaefer, T. R. Meyer, S. Roy, S. A. Danczyk, and J. R. Gord, Appl. Optics 48, B137 (2009).

[13] D. J. Kane and R. Trebino, Opt. Lett. 18, 823 (1993).

[14] J. Shan, A. S. Weling, E. Knoesel, L. Bartels, M. Bo nn, A. Nahata, G. A. Reider, and T. F. Heinz, Opt. Lett. 25, 426 (2000).

[15] Y. Kawada, T. Yasuda, H. Takahashi, and S. Aoshima, Opt. Lett. 33, 180 (2008).

[16] J. S. Robinson, C. A. Haworth, H. Teng, R. A. Smith, J. P. Marangos, and J. W. G. Tisch, Appl. Phys. B 85, 525 (2006).

[17] A. Suda, M. Hatayama, K. Nagasaka, and K. Midorikawa, Appl. Phys. Lett. 86, 111116 (2005). 
[18] R. V. Volkov, D. M. Golishnikov, V. M. Gordienko, P. M. Mikheev, A. B. Savel'ev, V. D. Sevast'yanov, V. S. Chernysh, and O. V. Chutko, JETP Lett. 72, 401 (2000).

[19] V. V. Yakovlev, Spectroscopy, 22, 34 (2007).

[20] B. L. Yu, A. B. Bykov, T. Qiu, P. P. Ho, R. R. Alfano, and N. Borelli, Opt.

Commun. 215, 407 (2003).

[21] B. C. Stuart, M. D. Feit, A. M. Rubenchik, B. W. Shore, and M. D. Perry, Phys. Rev. Lett. 74, 2248 (1995).

[22] G. M. Guss, I. L. Bass, R. P. Hackel, C. Mailhiot, and S. G. Demos, Appl. Optics 47, 4569 (2008). 


\section{Figure captions}

FIG 1: Probe signal as a function of gating beam delay using as the Kerr medium fused silica (black) and NSF-66 (red). The inset shows probe signal versus gating beam energy for the fused silica gate.

FIG 2: Gated measurement of a strong (7.5ps) and a weak (0ps) probe pulse. The dashed line indicates the background level caused by the strong pulse. The inset shows the weaker pulse measured with no strong pulse present (signal x 40).

FIG 3: Two-dimensional images of multiple-pulse irradiation of a glass target at a laser intensity $2.5 \times 10^{14} \mathrm{Wcm}^{-2}$ at a probe delay of $2.5 \mathrm{~ns}$ (a) without and (b) with optical gating. The left column shows the target after 10 shots; the right column after 100 shots.

FIG 4: Two-dimensional imaging of a wire through (a) zinc oxide wafer and (b) a sample of lead-bismuth-gallium glass. 


\section{Figure 1}

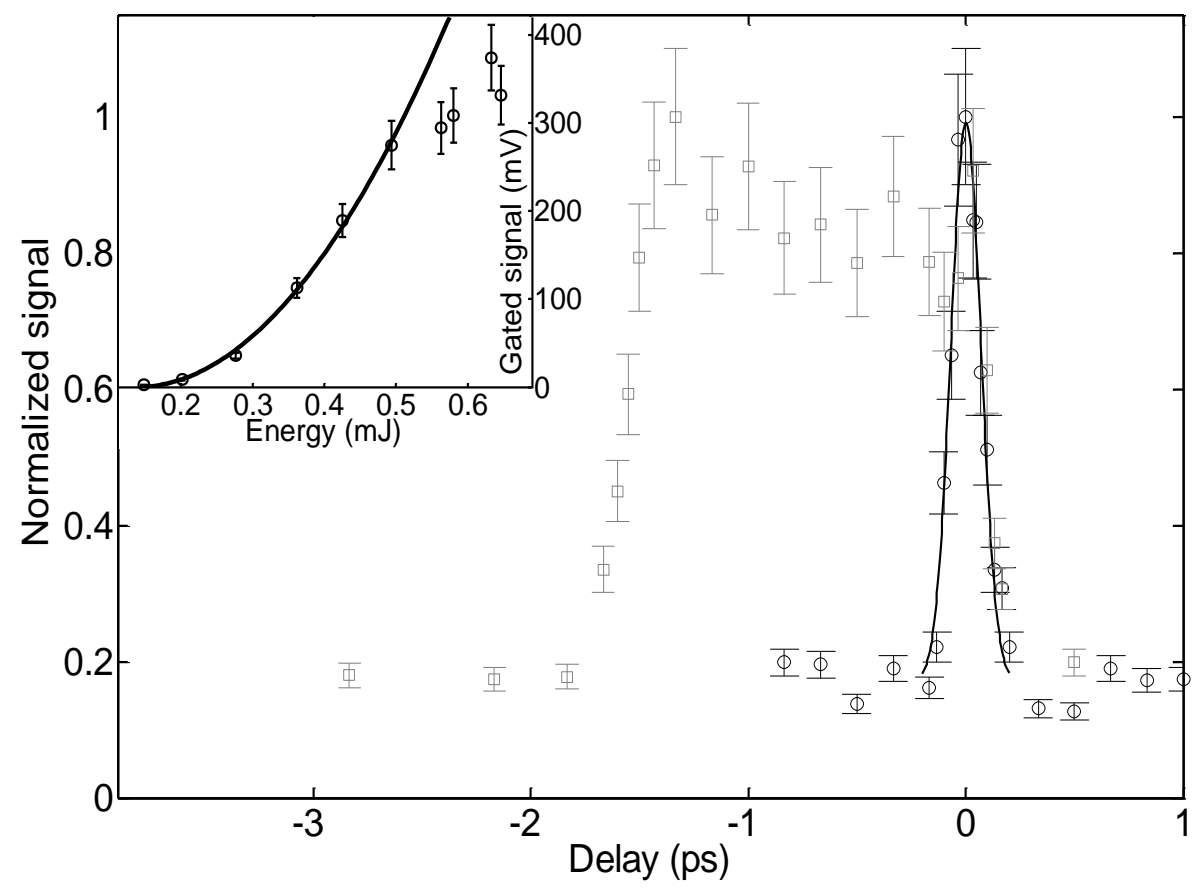

Figure 2

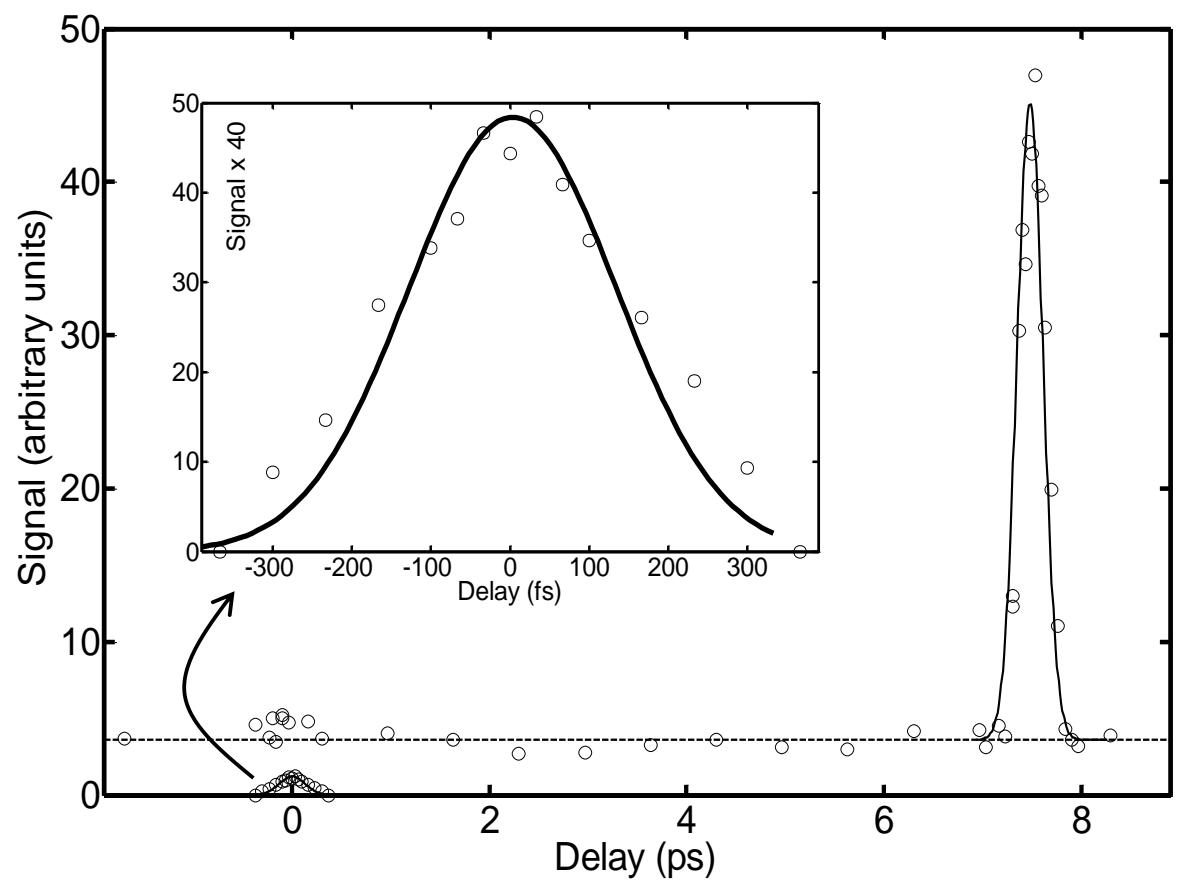




\section{Figure 3}
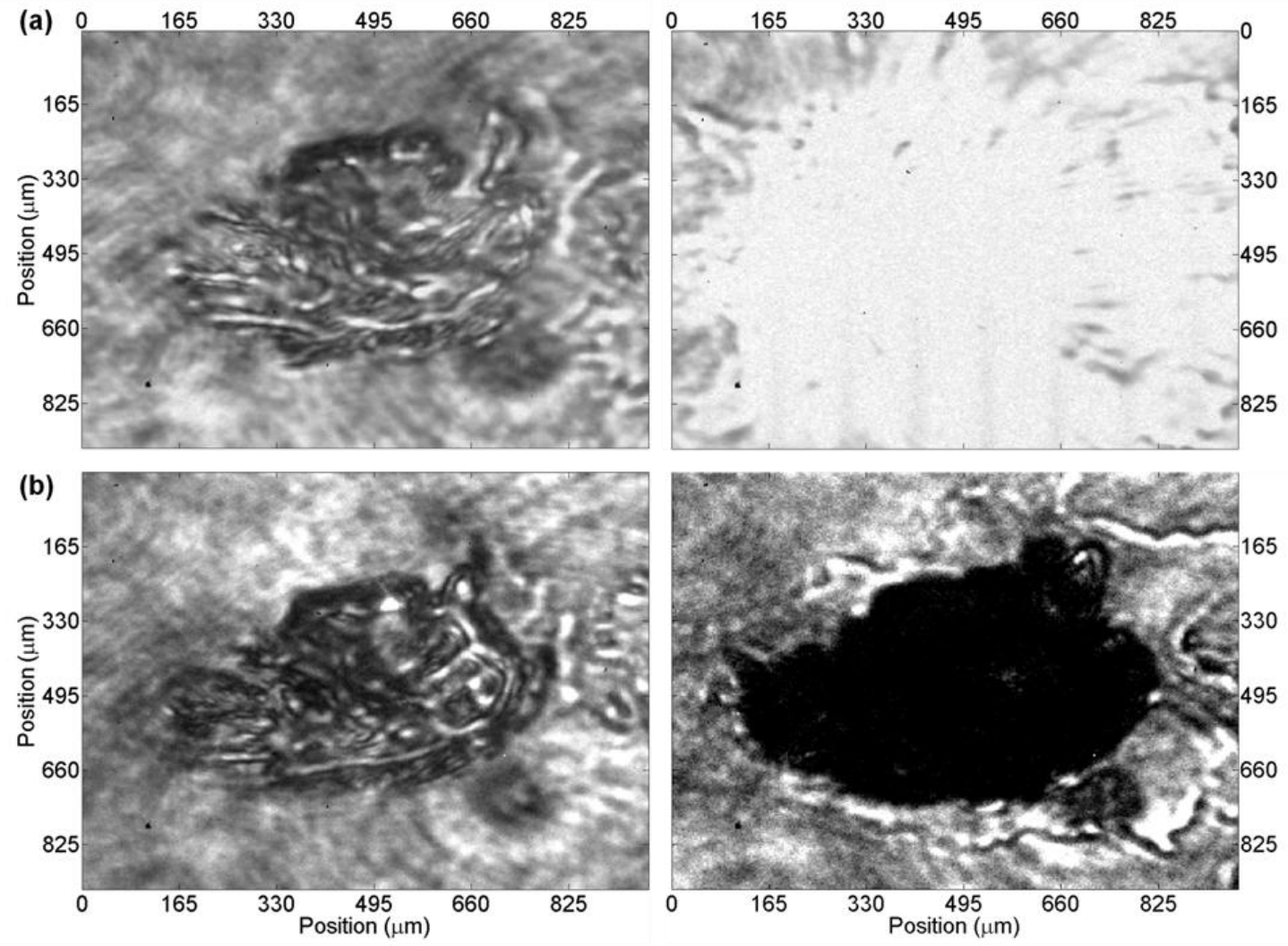

\section{Figure 4}
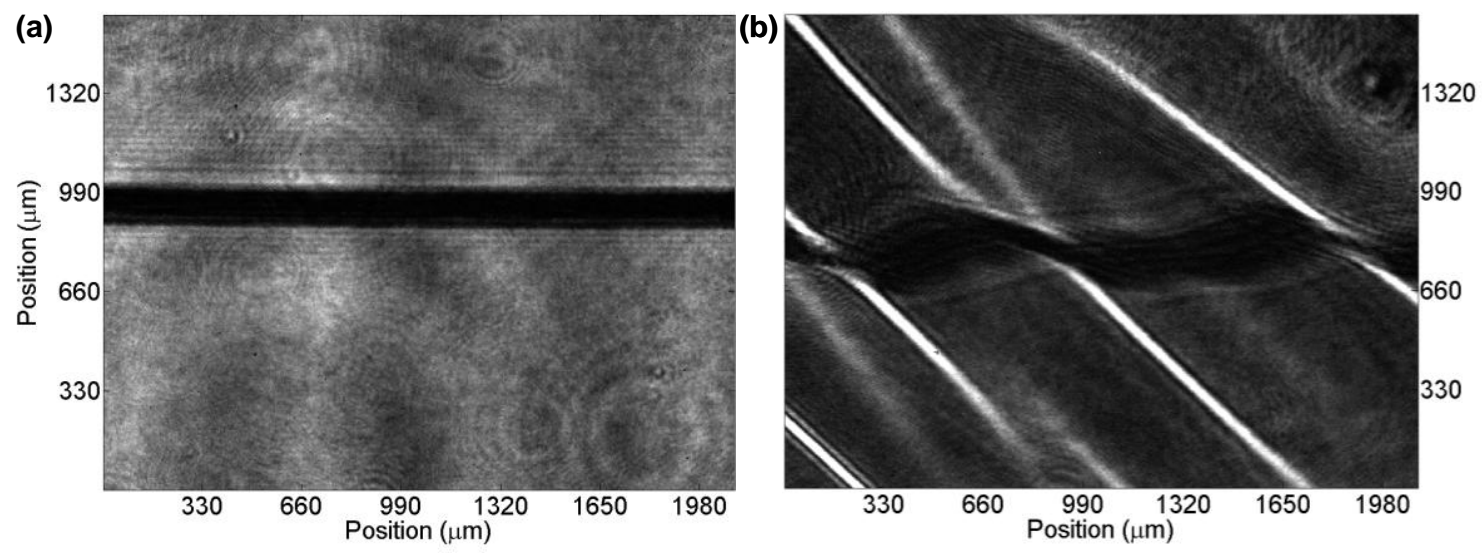